TABLEAU X

SGHÉMA DE STRUGTURE DE LA GASÉINE $\propto$ DE VACHE [I5]

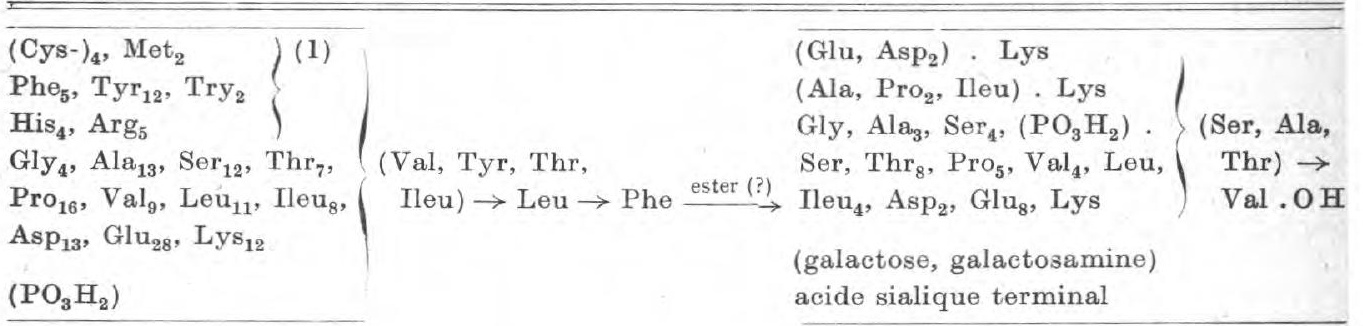

paracaséine $x$

caséino-glyeopeptide $x$

caséine $x$

(1) La totalité des acides aminés soufrés, aromatiques et basiques (sauf Lys) de la caséine $\%$ se trouvent dans la paracaséine $x$.

Le tableau $\mathrm{X}$ résume les précédentes données dans un schéma provisoire de la structure de la caséine $\chi$ (1962). L'existence possible d'une liaison ester entre la paracaséine $x$ et le caséino-glycopeptide sera discutée plus loin.

(A suivre.)

\title{
RECHERCHES SUR LA CORRÉLATION ENTRE LE POIDS SPÉCIFIQUE ET LA CONDUCTIBILITÉ ÉLECTRIQUE DU LAIT DE VACHE (1)
}

\begin{tabular}{llc}
\multicolumn{2}{c}{ par } \\
Nino PINO \\
Directeur & et & LUIGI CHIOFALO \\
& & Assistant, Chargé de Cours libre
\end{tabular}

Institut de zootechnie générale de la Faculté de médecine vétérinaire de l'Université de Messine

Dans le cadre des recherches qui ont été inscrites au programme de cet Institut sur le chapitre "Caractère naturel du lait ", nous avons entrepris la présente étude dans l'intention d'obtenir des

(1) Etude faisant partie du groupe de travaux exécutés avec les fonds du Ministère de l'Hygiène et de la Santé en Italie, Direction Générale des Services Vétérinaires. A ce Service et au Directeur Général des Services Vétérinaires actuel, M. le Professeur Iginio Altara, les auteurs adressent l'expression de leur très respectueuse reconnaissance. 
données toujours plus probantes visant à un diagnostic rationnel et facile des fraudes les plus diverses qui sont perpétrées quotidiennement au préjudice du lait. Pour de multiples raisons, ce dernier est l'aliment incriminé par excellence : un peu, comme il a déjà été dit bien des fois, en raison de son hétérogénéité physicochimique de constitution ; un peu parce qu'il est l'aliment typique, sinon exclusif, des âges les moins protégés; un peu parce qu'en raison de son prix très accessible il est l'aliment le plus consommé par les couches sociales les plus déshéritées; un peu enfin parce qu'il compte parmi les aliments possédant le plus vaste domaine d'applications diététiques.

Nous avons déjà mis l'accent [20] sur la variété et la complexité des fraudes tendant à déclasser et à dégrader tout ce qui constitue la particularité de la valeur biologique élevée du lait. Les fraudes et adultérations visant à masquer la soustraction d'éléments de valeur économique et biologique élevée (produits de remplacement), les fraudes et sophistications comportant l'emploi de substances étrangères de faible valeur économique et encore moins biologique, le plus souvent nocives pour la santé humaine, et le plus souvent dans l'intention principalement de maintenir dans le temps les qualités biologiques particulières du lait telles qu'elles existent immédiatement à sa production (conservateurs) ; fraudes et sophistications visant à masquer des états de débuts d'altérations organoleptiques ou pathologiques du lait (tromperie par addition de produits chimiques au lait).

L'un des instruments les plus traditionnels et, nous pourrions dire, les plus classiques pour dévoiler certaines fraudes et adultérations du lait consiste en la détermination de sa densité ou poids spécifique. De nombreux chercheurs ont insisté sur le fait que la mesure de la conductibilité électrique (cond. él.) est notablement constante (Beckmann, Thorner, Pino et Chiofalo, et autres) et que cette mesure est facile à exécuter dans la pratique pour déceler les fraudes et sophistications.

Déjà les premiers chercheurs qui se sont occupés de la conductibilité électrique du lait (DoHRMANn en 1889 et 1891, Thorner en 1891), avaient entrevu la possibilité de s'en servir pour la détermination du mouillage. BECKMANN est parvenu à la même conclusion, mais il a ajouté que la détermination de la conductibilité électrique se prêtait très bien à la recherche de neutralisants et conservateurs ajoutés au lait, même à doses infinitésimales. JoRDIS est du même avis. Lesage et Dongier ont trouvé utile la mesure de la conductibilité électrique pour la recherche du mouillage. Petersen est arrivé à l'affirmation selon laquelle la conductibilité 
électrique ne permettait pas d'établir avec certitude de petites adultérations par mouillage. BINAGHI, que l'on peut considérer avec raison comme un pionnier de la détermination de la conductibilité électrique du lait des différentes espèces animales, conclut que même un mouillage à 10 p. 100 peut être reconnu avec certitude par la mesure de la conductibilité électrique, et que celle-ci se révèle une méthode très sensible dans la recherche des conservateurs et neutralisants ajoutés au lait. Selon STROHEKER, la conductibilité électrique fournit quelque indication pour l'identification du mouillage, tandis que pour W URSTER elle est utile dans la recherche des "falsifications" du lait. ROEDER, à la suite de recherches approfondies, a conclu que la mesure de la conductibilité électrique ne peut servir à dévoiler le mouillage du lait, tandis qu'elle dénonce la présence de neutralisants et conservateurs. KRENN est du même avis. Les différents chercheurs (Schmid, Schrorf, Wurster, Rudiger et Mayr, Daunhofer et Moser, Krenn, Roeder, Stroheker, PopPe) sont d'accord pour affirmer que la mesure de la conductibilité électrique se prête très bien à la détection des laits anormaux, pathologiques, provenant de mamelles non saines, parce qu'ils sont très riches en chlorures, et ainsi KRENN soutient qu'une telle méthode devait même être préférée à la chlorimétrie, à l'épreuve de l'alizarol, "et même aux méthodes microscopiques et culturelles ».

Comme il est facile de le remarquer, grâce au panorama des données exposées, tous les chercheurs ne sont pas d'accord sur une variabilité de la conductibilité électrique à cause du mouillage du lait, tandis que tous sont d'accord sur le fait qu'elle peut être employée pour la recherche de neutralisants et conservateurs, et qu'elle peut donc se montrer très utile dans le domaine des fraudes et sophistications.

On sait que la détermination du poids spécifique (p.s.) constitue encore aujourd'hui l'instrument le plus facile et le plus immédiat pour la détection du mouillage du lait, bien qu'avec une certitude insuffisante. La possibilité de coupler ce procédé de recherche avec la mesure de la conductibilité électrique, notablement constante et précieuse révélatrice de l'équilibre dynamique du lait, a donc toujours sollicité l'attention de plusieurs chercheurs. KRENN écrit qu'une conductibilité électrique normale du lait implique aussi un poids spécifique et un indice de réfraction constants. MonteFREDINe et LA PortA, à la suite d'un vaste programme d'investigations sur le lait, concluent qu'il existe entre le poids spécifique et la conductibilité électrique une corrélation négative notablement élevée, et donc qu'à un poids spécifique faible doit correspondre 
une conductibilité électrique élevée et vice-versa. Si un faible poids spécifique est accompagné d'une faible conductibilité électrique, cela doit nous orienter vers la certitude d'un mouillage. C'est pourquoi, selon les mêmes auteurs, si la conductibilité électrique à elle seule ne peut permettre de détecter le mouillage, elle donne, associée à la détermination du poids spécifique, une réponse sûre pour mettre en évidence le mouillage du lait. La réunion des deux mesures pourrait donc permettre de détecter le mouillage $\mathrm{du}$ lait par des solutions salines, qui, dans certaines limites, laisseraient le poids spécifique inchangé, mais qui élèveraient notablement les valeurs de la conductibilité électrique au-dessus des valeurs normales.

Les développements qui précèdent sont suffisants pour souligner l'intérêt que revêt la mise au point des rapports qui interviennent entre la conductibilité électrique et le poids spécifique du lait, afin d'en mettre en évidence l'éventuelle corrélation statistique à la lumière de la méthodologie biométrique la plus récente, et d'en exprimer l'éventuelle connexion par des formules mathématiques d'interprétation non douteuse. Les présentes recherches voudraient en conséquence représenter une contribution à l'enrichissement des connaissances sur le diagnostic hygiénique et sanitaire du lait.

\section{Observations personnelles.}

Les 34 prélèvements de lait ont été effectués, avec les mesures de précautions convenables, dans les bidons qui sont habituellement transportés depuis les stations collectrices jusqu'à la Centrale (1).

Pour la mesure du poids spécifique, nous nous sommes servis du thermolactodensimètre de QUEVEnne, qui, comme on le sait, permet la détermination simultanée du poids spécifique et de la température. Nous nous sommes orientés vers l'emploi de cette méthode parce que c'est celle que l'on emploie ordinairement dans les inspections traditionnelles. Toutes les valeurs que nous avons obtenues sont les moyennes de quatre à cinq analyses.

Pour la détermination de la conductibilité électrique, nous avons adopté un mesureur électronique de la conductibilité, modèle LBR/B à pont de Wheatstone, construit par les Etablissements Weilheim (Haute-Bavière). Pour cette épreuve, le lait a été porté à la température de $20^{\circ} \mathrm{C}$. Toutes les mesures conducti-

(1) Nous tenons à remercier la Centrale Laitière de Messine, et en particulier, son directeur, M. le $D^{x}$ Vincenzo Antoci, pour leur extrême prévenance et cordialité. 
métriques ont été exécutées à cette température. Les valeurs, résultant chacune d'une moyenne de quatre à cinq épreuves, ont été exprimées en Siemens et indiquées par la notation $\lambda \times 10-4$ $(\lambda=$ valeur d'échelle), avant l'équation ci-jointe indiquée par nous dans nos précédents travaux sur la conductibilité électrique du lait.

Pour des raisons de place, nous laisserons de côté l'aspect des simples observations. Nous reproduisons au lieu de cela les paramètres les plus significatifs (tableau $n^{0} I$ ) afin d'en représenter synthétiquement l'évolution exprimée, comme on le sait, par les indices spécifiques relatifs : moyenne arithmétique, déviation, variation, sigma, erreur standard. Ce dernier indice, signifiant le degré de dispersion des moyennes probables se situant autour de celle qui est calculée, permet d'évaluer l'évolution de l'intensité des valeurs, fournissant des renseignements valables sur la confiance à accorder à la moyenne empirique. En outre, permettant le calcul des limites de confiance de la moyenne, cet indice permet d'établir l'intervalle entre lequel est identifiée la moyenne "vraie» ou "théorique » et donc d'apprécier les oscillations de la valeur typique de l'intensité, tant en ce qui concerne la conductibilité électrique que le poids spécifique.

Mais l'objectif des investigations était centré vers la recherche de l'éventuelle corrélation entre le poids spécifique et la conduc-

\section{TABLEAU I}

$\mathbf{x}=$ Poids spécifique du lait.

$\mathbf{y}=$ Conductibilité électrique du lait.

Limites de sécurité de la moyenne

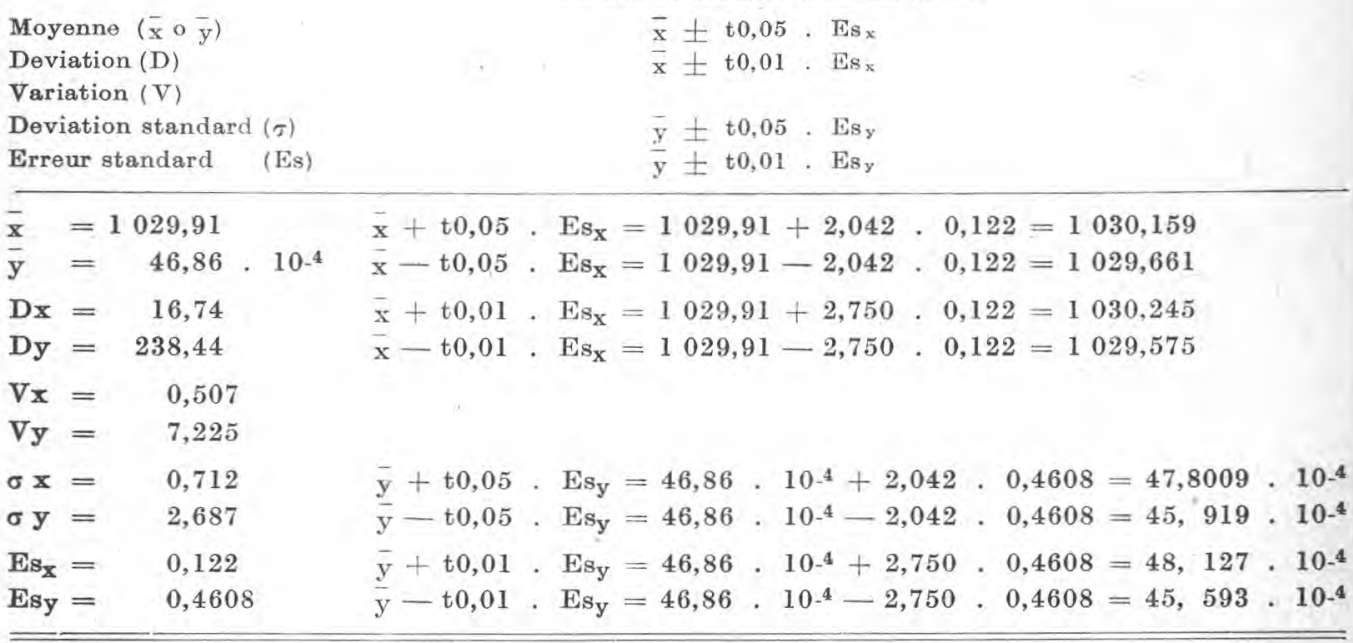


tibilité électrique du lait. C'est-à-dire vers l'essai de mettre en évidence une éventuelle dépendance statistique ou "stocastique " entre les deux importantes constantes physico-chimiques. II convenait cependant de calculer le coefficient de corrélation. Mais étant donné la valeur à attribuer à cet indice, nous nous sommes limités seulement à l'emploi de la formule de Bravais-Pearson, opportunément mise à jour comme on peut le lire dans un précédent travail de l'un de nous (1), mais, suivant les suggestions de la méthodologie la plus récente, nous avons recherché le coefficient de corrélation à travers le calcul de la codéviation et de la covariation, en l'absence du tableau de corrélation. En développant la formule de Bravais-Pearson, nous avons construit la table de corrélation avec les modifications que nous avons exposées du travail cité, afin de pouvoir obtenir un aspect signalétique le plus explicatif possible sur la distribution des fréquences, et de pouvoir apprécier rapidement, par cette représentation, l'existence ou au moins le signe de l'éventuelle corrélation. Pour le calcul de la codéviation, nous nous sommes done référés à la méthodologie suggérée dans ce travail (1), que nous rapportons brièvement pour plus de clarté :

$$
\begin{array}{ll}
\text { Dxy } & \begin{array}{l}
\text { (codéviation) } \\
\text { Vxy }
\end{array} \\
\mathrm{r} & \begin{array}{l}
\text { (covariation) } \\
\text { (coeficient de corrélation) }
\end{array} \\
\mathrm{N} & \text { (nombre de couples) } \\
\mathrm{x} & \text { (poids spécifique) } \\
\mathrm{y} & \text { (conductibilité électrique) } \\
\mathrm{Dxy}= & \mathrm{\Sigma x} \cdot \mathrm{y}-\mathrm{Tx} \cdot \mathrm{Ty} / \mathrm{N} \\
& \mathrm{Vxy}=\frac{\mathrm{Dxy}}{\mathrm{N}-\mathrm{I}} \\
& \mathrm{r}=\frac{\mathrm{Vxy}}{\sigma \mathrm{x} \cdot \sigma \mathrm{y}}
\end{array}
$$

En observant le tableau de corrélation (tableau II), on relève qu'il n'existe pas de corrélation entre le poids spécifique et la conductibilité électrique des échantillons prélevés. En effet, la distribution des fréquences dans les quatre compartiments rectangulaires est quelque peu irrégulière et désordonnée, tandis que l'on sait que la corrélation entre deux variantes - dans notre cas entre le poids spécifique et la conductibilité électrique —, "qu'elle soit

(1) Luigi Chiofalo. Corrélation entre le poids de l'œuf et le poids du poussin à la naissance. Recherches biométrico-statistiques à la lumière de la méthodologie moderne. Zootecnia e Vita, Anno II, no 1, 1959. 
TABLEAU II

TABLEAU DE GORRÉLATION

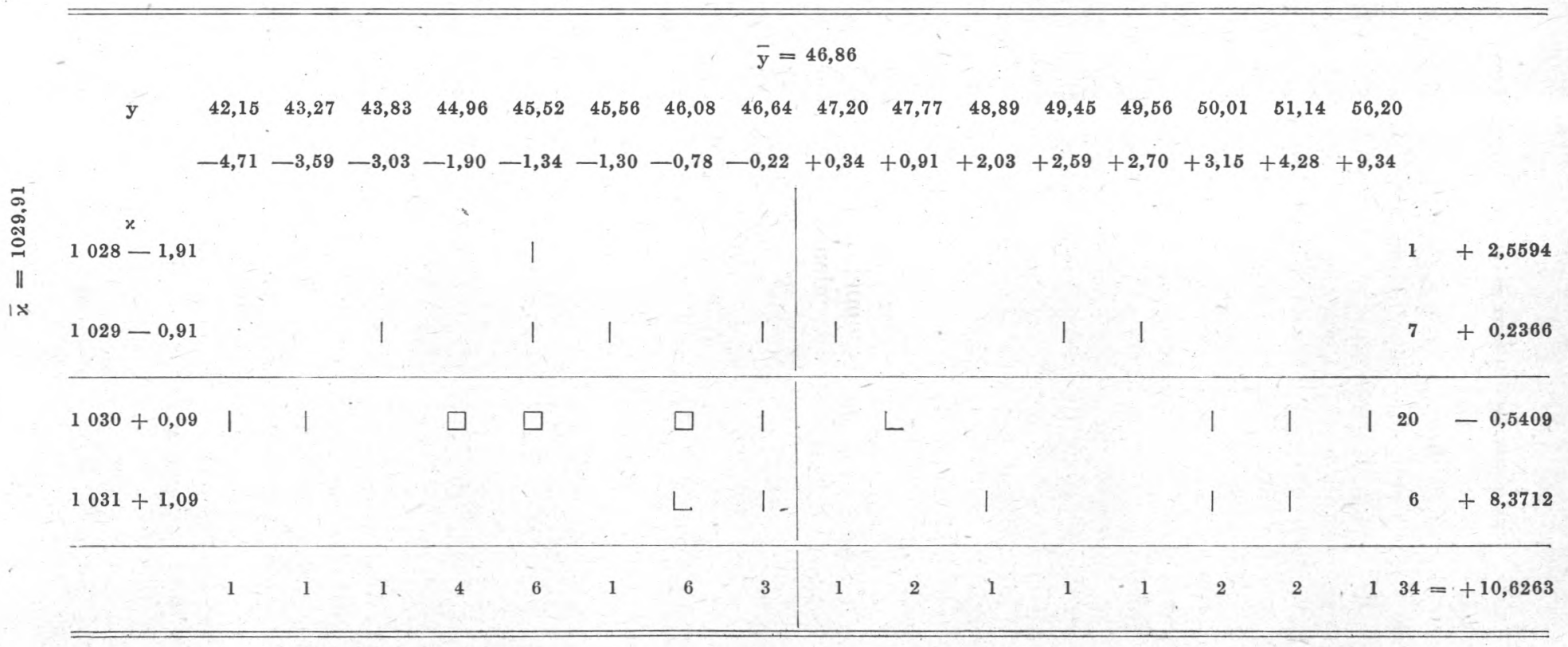


positive ou négative, est témoignée par la concentration simultanée et ordonnée des fréquences dans deux compartiments distincts du tableau opposé par le sommet, c'est-à-dire au point d'origine des coordonnées, qui délimitent les quatre compartiments. C'est précisément une corrélation positive qui est exprimée par le cumul des fréquences dans les compartiments supérieur gauche et inférieur droit; la corrélation négative est indiquée par la situation inverse : gravitation de fréquences dans les compartiments inférieur gauche et supérieur droit. Il n'est pas possible de mettre en évidence aucune de ces deux conditions dans notre tableau de corrélation.

La non-existence de la corrélation, entre le poids spécifique et la conductibilité électrique du lait, est montrée encore plus clairement par le calcul du coefficient de corrélation. Comme nous l'avons déjà dit, étant donné l'importance que revêt la question, nous avons recherché le coefficient de corrélation entre le poids spécifique et la conductibilité électrique en suivant deux méthodes bien distinctes : toutes les deux ont conduit à deux résultats identiques et superposables. En effet, $r$ (coefficient de corrélation) est égal à 0,168 tant dans le premier cas que dans le second cas (tableau no II). Ceci apporte non seulement le témoignage de la justesse de la méthodologie suivie, mais encore contribue à approfondir le sujet proposé par nous et, nous l'affirmons, peut nous autoriser à évaluer avec tranquillité les résultats obtenus.

Le coefficient calculé, précédé du signe $(+)$, témoignerait à première vue en faveur d'une corrélation positive, bien que très faible, entre le poids spécifique et la conductibilité électrique de notre échantillon. Mais pour que l'on puisse prendre en considération un tel coefficient, il est nécessaire d'en vérifier la sécurité ou l'importance. Pour cette procédure également, afin d'en garantir le maximum, nous avons effectué diverses épreuves et précisément : 1) celle consistant à comparer le coefficient obtenu avec l'erreur propre ; 2) celle consistant à rapporter le même coefficient aux niveaux de sécurité de Fisher-Yates ; 3 ) celle du " $t$ » de Fischer.

Les résultats de ces épreuves ont été assez indicatifs (tableau n० III)

Première épreuve : l'erreur du coefficient de corrélation, obtenue selon Fisher, est égale à la valeur du coefficient de corrélation; la même erreur, recherchée selon VIANELLI, est presque égale à cette valeur. Or, afin que le coefficient de corrélation puisse être sûr c'est-à-dire significatif, il doit dépasser de quatre à cinq fois sa propre erreur.

Conclusion : aucune garantie de signification. 
Deuxième épreuve : la valeur du coefficient de corrélation ne dépasse pas les niveaux de sécurité, tant pour $\mathrm{P}=0,05$ que pour $P=0,01$, et elle est donc très inférieure.

Conclusion : il se confirme que le coefficient obtenu n'est ni significatif ni sûr.

Troisième épreuve : le $t$ calculé est égal à 0,962 , tandis que le $t$ du tableau, tant à 5 p. 100 qu'à 1 p. 100, est très supérieur, comme on peut le constater au tableau no II.

\section{TABLEAU III}

$$
\begin{aligned}
& D x y=10,63 \\
& V x y=0,322 \\
& r \quad=0,168 \\
& E r=\frac{1-r^{2}}{\sqrt{\bar{N}-1}}=\frac{1-0,168^{2}}{\sqrt{33}}=0,168 \quad \text { (Fisher) } \\
& E r=\frac{1-r^{2}}{\sqrt{\bar{N}}}=\frac{1-0,168^{2}}{\sqrt{34}}=0,166 \quad \text { (Vianelli) }
\end{aligned}
$$

Niveaux de sécurité (tableau de Fisher-Yates)

Pour 30 paires :

$$
\begin{aligned}
& 0,3494 \text { per } \mathrm{P}=0,05 \\
& 0,4487 \text { per } \mathrm{P}=0,01
\end{aligned}
$$

tde Fisher :

$t=\frac{\mathrm{r}}{\sqrt{1-\mathrm{r}^{2}}} \cdot \sqrt{\mathrm{N}-2}=\frac{0,168}{\sqrt{1-0,168^{2}}} \cdot \sqrt{32}=\frac{0,168}{0,985} \cdot 5,656=0,962$

ipour 30 degrés d'écart :

$$
\begin{aligned}
& 2,042 \text { per } \mathrm{P}=0,05 \\
& 2,750 \text { per } \mathrm{P}=0,01
\end{aligned}
$$

$x \quad=$ Poids spécifique du lait.

$y=$ Conductibilité du lait.

$D x y=$ Codéviation.

$\nabla x y=$ Covariation.

$r \quad=$ Coefficient de corrélation.

$E r \quad=$ Erreur du coefficient de corrélation. 


\section{Considérations conclusives.}

Les diverses épreuves de signification ou de reproductibilité des résultats ont montré que le coefficient de corrélation entre le poids spécifique et la conductibilité électrique du lait, dans les limites de nos recherches, n'est pas important. En nous tenant néanmoins aux résultats, il ne s'est manifesté aucune dépendance statistique entre les deux caractéristiques physico-chimiques. A notre avis, cette découverte trouverait quelque justification dans l'examen du substratum déterministique des deux constantes. En ce qui concerne le poids spécifique du lait, il semble tout naturel d'admettre, comme il a été synthétisé par Mantovani, qu'il dépend de la teneur en matière grasse et en extrait sec dégraissé (protides, lactose, sels). La matière grasse agirait dans le sens de la diminution du poids spécifique du lait, l'extrait sec manifesterait une influence opposée. La conductibilité électrique du lait est en fonction de sa concentration ionique : nous en trouvons encore confirmation chez MaNTovanI et cela semble être tout naturel également pour tous les chercheurs qui s'en sont occupés. Des substances à l'état de solutions moléculaires, suspensoïdes et colloïdes (Mantovani, Gerber, Flohil, Jaokson et Rothera, et autres), manifesteraient une action très nette déprimante sur la conductibilité électrique. Il y aurait donc un substratum déterministique nettement différent pour les deux constantes. Du reste, les quelques références bibliographiques démontrent l'unanimité de vue au sujet de l'action révélatrice de la conductibilité électrique dans les cas d'addition au lait de produits conservateurs, neutralisants, et dans le diagnostic de laits provenants de mamelles pathologiques, mais elles ne démontrent pas autant d'unanimité au sujet de l'action de la conductibilité électrique dans la détection du mouillage du lait.

C'est pourquoi on manquerait de base pour une relation quelconque, ou mieux une corrélation, entre le poids spécifique et la conductibilité électrique du lait. A ce sujet, Petersen est assez explicite lorsqu'il exclut nettement une quelconque relation entre les deux constantes; même si KRENN se borne à affirmer que, pour des laits à conductibilité électrique normale, il correspond un poids spécifique et un indice de réfraction normaux.

Les résultats auxquels sont parvenus Montefredine et $\mathrm{L}_{\mathrm{A}}$ Porta, selon lesquels il existerait entre le poids spécifique et la conductibilité électrique du lait une corrélation négative notablement élevée, seraient manifestement en contradiction avec ce qui précède. Nous ne croyons pas qu'une telle corrélation négative 
notablement élevée, pour ne pas dire très élevée (la valeur du coefficient trouvé par Monterredine et LA Porta est de - 0,9043) entre les deux constantes examinées puisse s'expliquer à la lumière des connaissances actuelles sur le mécanisme déterministique $d u$ poids spécifique et de la conductibilité électrique. Il n'est pas inopportun de répéter en effet que la matière grasse, qui, parmi les constituants du lait joue un rôle de premier ordre du point de vue physico-chimique, manifeste tant sur le poids spécifique que sur la conductibilité électrique une nette action déprimante unanimement reconnue. Sous cet aspect, done, la corrélation devrait être positive parce qu'un lait riche en matière grasse devrait avoir un poids spécifique et une conductibilité électrique caractérisés par de faibles valeurs. D'autre part, l'extrait sec dégraissé, le lactose et les protides en particulier, selon la majorité des chercheurs, manifesteraient une action négative sur la conductibilité électrique du lait, tandis qu'ils feraient augmenter les valeurs du poids spécifique : sous cet autre aspect, on pourrait donc entrevoir une corrélation négative entre les deux constantes physico-chimiques. Il faut ajouter en outre que si les substances développent à l'état ionique une action très nette et univoque pour faire augmenter les valeurs de la conductibilité électrique du lait, ces mêmes substances manifestent un rôle très modeste dans le déterminisme du poids spécifique. En effet, la diminution du poids spécifique du lait de mammite, due surtout à la diminution de la teneur en lactose, ne parvient pas à être contre-balancée par l'augmentation de la teneur en $\mathrm{NaCl}$ (MANTovani), qui, par contre, est très efficace pour faire augmenter notablement les valeurs de la conductibilité électrique.

La plateforme de conditionnement des valeurs respectives nous semble pour cela assez complexe ainsi que différenciée dans sa dynamique. Il faudrait en exclure - en considérant le lait comme un semi-tissu (ANTONIANI), un semi-tissu polyphasique, pourrions-nous dire - un jeu de polarités et de contre-balancement entre positivité et négativité des deux corrélations. Le point réconfortant est que soit admis, comme nous l'avons déjà précisé, le rôle divers et contrastant manifesté par les iono-dispersoïdes en ce qui concerne les deux constantes. Nous aurons ainsi les trois schémas de corrélation hypothétiques suivants :

Matière grasse $<$ poids spécifique Solutions moléculaires et collotdes coids spécifique 


\section{Substances ioniques $>$ poids spécifique (non important)}

La dynamique d'un substratum déterministique aussi hétérogène et polymorphe pourrait conditionner des domaines de gravitation qui n'autoriseraient pas à admettre l'existence statique d'une corrélation entre les deux constantes, de quelque signe qu'elle soit; moins encore, d'une corrélation très élevée.

\section{RÉSUME}

Les auteurs, dans le cadre des recherches entreprises dans l'intention d'apporter une contribution au diagnostic hygiénique et sanitaire sur le "caractère de pureté naturelle du lait ", ont étudié la corrélation entre le poids spécifique et la conductibilité électrique du lait de vache. Les recherches correspondantes ont permis de conclure qu'il n'existait pas de corrélation quelconque entre les deux constantes physico-chimiques.

Les résultats sont commentés à la lumière des plus récentes connaissances acquises sur les substances qui conditionnent les valeurs densimétriques et conductimétriques du lait et sur la physico-chimie moléculaire.

\section{SUMMARY}

In order to concur in the study of the sanitary, hygienic diagnosis regarding "the standard of natural purity of milk ", the authors have observed the correlation between the specific gravity and the electrical conductivity of cows' milk : there is not any correlation between these two constants.

They comment upon the results by the light of the last knowledge that has been gained concerning, first, the substances that condition the specific gravity and the electrical conductivity of milk, and, second, the molecular physics and chemistry.

\section{BIBLIOGRAPHIE}

[1] Beckmann. Cité par Montefredine A.

[2] R. Binaghr. Biochem. Zeit., 1910, t. XXIX, p. 60.

[3] L. Chrofalo. Leçons au cours de biométrie et de méthodologie biométrique. Zootecnia e Vitâ, 1959, II année, nos 2-3 et no 4.

[4] L. Chiofalo. Leçons au cours de biométrie et de méthodologie biométrique. Zootecnia e Vitâ, 1961, IVe année, no 4. 
[5] Daunhofer et Moser. Cités par A. Montefredine.

[6] Dohrmann. Cité par W. Kopaczewski. Physico-Chimie du Lait. Ed. Dunod, Paris, 1950 .

[7] R. A. Fisher. Méthodes statistiques à l'usage des chercheurs. U.T.E.T., Turin, 1948.

[8] R. A. Fisher et F. YATES. Tableaux statistiques pour la recherche biologique, agronomique et médicale. Edimbourg et Londres, 1949, Oliver et Boyd.

[9] Flohil. Cité par A. Montefredine.

[10] V. Gerber. Cité par F. Pinkerton et I. I. Peters. Conductibilité, teneur pour cent en lactose et point de congélation du lait. Journal of Dairy Science, 1958, vol. 41, n ${ }^{\circ} 3,392 \mathrm{p}$.

[11] L. C. Jackson et A. C. H. Rothera. Cité par E. Pinkerton et I. I. Peters.

[12] Tordis. Cité par A. Montefredine.

[13] J. KRENN. Valeur de la conductibilité du lait pour l'estimation chimique et hygiénique de celui-ci. Le Lait, 1933, t. XIII, 922 p.

[14] Lesage et Dongier. C. R. Acad. Sc., 1902, t. CXXXIV, p. 612.

[15] G. Mantovani. Inspection des aliments d'origine animale, U.T.E.T., Turin, 1961.

[16] A. Montefredine. La conductibilité électrique comme moyen de recherche dans l'analyse du lait. Annali di Chimica Applicata, 1942 , vol. 32 , fasc. 2 .

[17] A. Montefredine et L. La Porta. La conductibilité électrique du lait. Atti XIVe Congrès International du Lait et Dérivés, 1956, vol. III.

[18] A. Niceforo. Cité par F. Usuelli. Du génotype et des problèmes inhérents de zootechnie générale. Cisalpino, Milan.

[19] Petersen. Thèse de Kiel, 1904.

[20] A. Pino et L. Chiofalo. Recherches sur la conductibilité électrique du lait de chèvre. Zootecnia e Vita, 1962, Ve année, no 4. Le Lait, 1963 , t. XLIII, nos $429-430$, p. 603.

[21] A. Pino et L. Chiofalo. Modifications de la viscosité, de la tension superficielle et de la conductibilité électrique du lait à la suite du transport. Le Lait, 1963, t. XLIII, nos 421-422, p. 2.

[22] Poppe. Cité par A. Montefredine et L. La Porta.

[23] G. Roeder. Recherches sur la conductibilité électrique du lait. Le Lait, 1932, t. XII, p. 342.

[24] Rugiger, Wurster et Mayr. Cités par A. Montefredine.

[25] Schmid. Cité par A. Montefredine.

[26] Schnorf. Cité par A. Montefredine.

[27] Strohecker. Cité par A. Montenredine.

[28] Thorner. Cité par A. Montehredine.

[29] Wurster. Cité par A. Montefredine. 